\title{
Treatment options for vasomotor symptoms in menopause: focus on desvenlafaxine
}

\author{
This article was published in the following Dove Press journal: \\ International Journal of Women's Health \\ 4 July 2012 \\ Number of times this article has been viewed
}

\author{
Elena M Umland \\ Laura Falconieri \\ Jefferson School of Pharmacy, \\ Thomas Jefferson University, \\ Philadelphia, PA, USA
}

Correspondence: Elena M Umland Jefferson School of Pharmacy, Thomas Jefferson University, 90I Walnut Street, Suite 90IB, Philadelphia, PA 19107, USA

$\mathrm{Tel}+$ I 2155039087

Fax +I 2155039052

Email elena.umland@jefferson.edu

\begin{abstract}
Vasomotor symptoms (VMS), including hot flashes and night sweats, occur in as many as $68.5 \%$ of women as a result of menopause. While the median duration of these symptoms is 4 years, approximately $10 \%$ of women continue to experience VMS as many as 12 years after their final menstrual period. As such, VMS have a significant impact on the quality of life and overall physical health of women experiencing VMS, leading to their pursuance of treatment to alleviate these symptoms. Management of VMS includes lifestyle modifications, some herbal and vitamin supplements, hormonal therapies including estrogen and tibolone, and nonhormonal therapies including clonidine, gabapentin, and some of the serotonin and serotonin-norepinephrine reuptake inhibitors. The latter agents, including desvenlafaxine, have been the focus of increased research as more is discovered about the roles of serotonin and norepinephrine in the thermoregulatory control system. This review will include an overview of VMS as they relate to menopause. It will discuss the risk factors for VMS as well as the proposed pathophysiology behind their occurrence. The variety of treatment options for VMS will be discussed. Focus will be given to the role of desvenlafaxine as a treatment option for VMS management.
\end{abstract}

Keywords: menopause, vasomotor symptoms, hot flashes, vasomotor symptom treatment, desvenlafaxine

\section{Introduction}

The final menstrual period (FMP) marks the point in time between the menopausal transition and postmenopause. ${ }^{1}$ Natural menopause, specifically, is confirmed after 12 consecutive months of amenorrhea in the absence of any obvious, pathologic cause. ${ }^{2}$ These 12 months of amenorrhea, inclusive, characterize a woman as postmenopausal. ${ }^{1}$ This can further be divided into early postmenopause (1-4 years after the FMP) and late postmenopause ( $>5$ years since the FMP). ${ }^{1,3}$ Surgical menopause results following surgical removal of the ovaries. In either instance, one result of the declining estrogen concentrations is the occurrence of vasomotor symptoms (VMS) that include hot flashes and night sweats.

VMS of any degree, mild to severe, are experienced by $58.9 \%$ of Latin American women at perimenopause, the years immediately surrounding the $\mathrm{FMP}^{3}$ The peak incidence of VMS is noted to occur in American women 1 year after the FMP, ${ }^{4}$ and $68.5 \%$ of women experience VMS of some degree in early menopause, which is described as $1-5$ years after the FMP. ${ }^{3}$ Further, the median duration of VMS occurrence is 4 years, while approximately $10 \%$ of women experience VMS as long as 12 years after the FMP. ${ }^{4}$ submit your manuscript | www.dovepress.com

Dovepress

http://dx.doi.org/10.2147/IJWH.S24614 
Severe VMS occurs in $10.8 \%$ of women in the perimenopausal time frame, in $12.3 \%$ of women during early menopause, and in $11.5 \%$ of women in late menopause, defined as greater than 5 years from the FMP. ${ }^{3}$ The prevalence and severity of VMS is greater in women who are surgically menopausal compared to women experiencing natural menopause. ${ }^{3}$ Given the overall frequency of these symptoms, it is important to be aware of the risk factors for their occurrence such that if the risk factor(s) are modifiable, the risk of VMS could be reduced. Further, awareness of risk may help to identify those women who may be in greatest need of VMS treatment as a means to improve quality of life (QOL) and overall health.

\section{VMS risk factors}

Several risk factors for the occurrence and severity of VMS have been established. ${ }^{5-7}$ Obesity, race, and smoking have been most closely associated with the occurrence of VMS. Various reports of the linkage between obesity and VMS include the fact that VMS were observed to be absent or infrequent in a population of women with a mean body mass index (BMI) of $28 \mathrm{~kg} / \mathrm{m}^{2}$ while women with an average BMI of $31 \mathrm{~kg} / \mathrm{m}^{2}$ were more likely to report VMS as occurring more frequently than their lower-BMI counterparts. ${ }^{5}$ Further, Gold and colleagues discovered similar findings as the frequency of VMS was statistically associated with the woman's BMI $(P<0.0001)$; the average BMI for women experiencing no VMS or symptoms for $<6$ days in the 2 weeks prior to evaluation was $28 \mathrm{~kg} / \mathrm{m}^{2}$, while the average BMI for women experiencing VMS $\geq 6$ days in the prior 2 weeks was $30.9 \mathrm{~kg} / \mathrm{m}^{2,6}$ Specifically, another study established a sevenfold greater risk for being in the highest quartile of having the most bothersome VMS for every $2.7 \mathrm{~kg} / \mathrm{m}^{2}$ increase in BMI. ${ }^{7}$ While the physiologic relationship between BMI and VMS frequency and severity is not completely understood, it is thought to be linked to the role of adipose tissue as an insulator that prevents the release of heat. ${ }^{5}$

Race and ethnic variations are also associated with differences in the incidence of VMS. One study established that the frequency of VMS is significantly greater among African American women compared to women of other races $(P<0.01) .{ }^{6}$ While the exact reason for this is unknown, this population of women has been observed to have the highest BMIs and highest rates of current smoking and exposure to tobacco smoke. Further, African American women have been observed to exhibit a different perception and tolerance of VMS compared to other racial and ethnic populations.
Another study confirmed this finding in African American women when it reported that they are most likely to report and describe their VMS as bothersome. ${ }^{5}$ Caucasian and Hispanic women report VMS as often as African American women, but do not report them to be as severe. Chinese and Japanese women are the least likely to report VMS or to describe them as bothersome. ${ }^{5}$ Overall, variability among different cultures may be related to variability (by culture) of other determinants of VMS, such as hormone use, age, BMI, depression, anxiety, poor physical health, perceived stress, cigarette smoking (and exposure to passive smoke), and acculturation. ${ }^{8}$

A link between cigarette smoking as well as passive exposure to tobacco smoke and the occurrence of VMS has been hypothesized to exist, as cigarette smoking may exhibit antiestrogenic effects. Study findings have illustrated that smokers are $60 \%$ more likely to report any VMS compared to nonsmokers. ${ }^{5}$ Additionally, the frequency of VMS is associated with current smoking; the odds ratio for smokers reporting VMS being 1.63 (range $1.25-2.12 ; P<0.01$ ) compared to nonsmokers. ${ }^{6}$

Other risk factors for VMS that have been identified but for which the data are limited include physical inactivity, the presence of negative affect or anxiety, and educational status. The associations between physical inactivity and VMS have been studied and described as weak. ${ }^{5}$ While anxiety and negative affect have been associated with VMS occurrence and frequency as well as an increased likelihood of the VMS being described as bothersome, ${ }^{5}$ these findings have not been studied extensively. In fact, it is difficult to reach any conclusion using the available studies that would be applicable to practice, as the studies utilized poor, largely nonvalidated measures of said symptoms. ${ }^{9}$ Similarly, an association between VMS and level of education has been observed. VMS reports have been observed to be higher among women with some college education compared to women with high school as their terminal level of education increases. ${ }^{6}$ This has not been replicated or reported elsewhere.

\section{Impact of VMS on quality of life}

VMS negatively affect QOL,, 3 and this has been evaluated globally as well as specifically for its impact on the factors contributing to QOL. These factors include sexual function, sleep quality, mood, and cognitive function.

The poorest health-related QOL has been observed among women with the most frequent VMS. ${ }^{5}$ More precisely, when comparing women who experience VMS to those in whom VMS are absent, any degree of VMS is associated with 
nearly a fivefold higher risk for severe QOL impairment; severe VMS are associated with a 20-fold higher risk for severe QOL impairment ( $P=0.0001$ for both compared to absent VMS). ${ }^{3}$ Women experiencing any degree of VMS are at an increased risk of experiencing other menopausal symptoms when compared to women who do not have VMS. Specifically, in women who experience VMS, $62 \%$ of them also experience sexual disturbances, $74 \%$ suffer from sleep disturbances, $76 \%$ note the occurrence of depressed mood, and $78 \%$ experience increased mental exhaustion. These menopausal symptoms occur in $29 \%, 41 \%, 42 \%$, and $49 \%$ of women without VMS, respectively. ${ }^{3}$ Further, Thurston and Joffe's findings also concluded that the presence of VMS is associated with all aspects of sleep disturbances including poor continuity and quality and early morning awakening. ${ }^{5}$

\section{VMS pathophysiology}

VMS are theorized to result from dysfunction in the woman's "tightly controlled temperature circuitry," leading to exaggerated activation of heat-dissipation responses such as peripheral vasodilation and sweating. ${ }^{10}$ The thermoregulatory circuitry includes the brain, internal body cavity, and the peripheral vasculature. It is thought that this circuitry functions under the influence of consistent concentrations of the neurotransmitters serotonin and norepinephrine.

Changes in gonadal hormones, notably estrogen, have been associated with fluctuations in serotonin and norepinephrine. The occurrence of VMS with the fluctuating levels of estrogen observed during perimenopause and the further declining levels of estrogen as occurs after menopause is likely influenced by the resultant fluctuating levels of serotonin and norepinephrine, contributing to disruption of the thermoregulatory circuitry. ${ }^{10}$ Specifically, reduced estrogen concentrations within the hypothalamic temperatures center negatively affect serotonin and norepinephrine concentrations in this location and contribute significantly to the occurrence of VMS. ${ }^{11}$ Temperature information is processed within the anterior hypothalamus in the preoptic area (POA), where it is felt that heat-sensitive neurons control the release of heat. ${ }^{11}$ Serotonergic neurons and noradrenergic pathways have both been identified as projecting into the POA. As the POA is directly related to temperature control and under the influence of serotonin and norepinephrine, the fluctuating estrogen concentrations that exist with menopause lead to VMS.

Given the data linking estrogen fluctuations to a reduction of serotonin and norepinephrine, it has been postulated that pharmacologic agents such as serotonin reuptake inhibitors and serotonin-norepinephrine reuptake inhibitors may help to reestablish levels of these neurochemicals within the thermoregulatory centers, reducing the occurrence of VMS. ${ }^{10,11}$

\section{VMS treatment Nonpharmacologic}

Many nonpharmacologic treatments have been identified to treat VMS. These include but are not limited to acupuncture, hypnosis, and lifestyle changes. The overall evidence supporting these treatments is variable. See Table 1 for a list of potential nonpharmacologic treatment options and a summary of the evidence available for each.

\section{Pharmacologic}

\section{Herbals/vitamins}

A variety of herbal medications and vitamin supplements have been studied for the treatment of VMS associated with menopause, including black cohosh, dehydroepiandrosterone, evening primrose oil, phytoestrogens, and vitamin E. Similar to the findings for the nonpharmacologic interventions for VMS, the efficacy findings for herbal medications and

Table I Nonpharmacologic treatments for vasomotor symptoms (VMS) $)^{12-18}$

\begin{tabular}{ll}
\hline $\begin{array}{l}\text { Nonpharmacologic } \\
\text { treatment }\end{array}$ & Evidence \\
\hline Acupuncture & - A randomized clinical trial illustrated that acupuncture was as effective as venlafaxine at reducing hot flushes. ${ }^{12}$ \\
& - Another study and review have shown a significant reduction in the mean number of hot flushes with acupuncture \\
& compared to placebo. ${ }^{13,14}$ \\
- Typnosis & Two small studies in breast cancer patients showed that hypnosis was more effective at reducing vasomotor \\
- Layered clothing & - Studies have shown that keeping cool by using layered clothing, cooler room temperatures, drinking cool liquids, and \\
- Cool atmosphere & - Studies regarding the efficacy of exercise in relieving VMS are ongoing; ${ }^{18}$ however, at this time there is an overall \\
- Cool drinks & lack of randomized controlled clinical trials. \\
- Avoiding hot/spicy food & - Clinical trials evaluating the effect of weight loss on VMS are lacking. \\
- Exercise/weight loss & - Many researchers believe smoking cessation can help reduce vasomotor symptoms, although evidence in this area is \\
- Smoking cessation & lacking, as there are currently no clinical trials evaluating this idea. \\
\hline
\end{tabular}


vitamin supplements are also inconsistent. ${ }^{19}$ Further, the longterm safety of these products is unclear. ${ }^{19}$ Table 2 depicts the various herbal medications and vitamins that have been examined for menopausal-related VMS, including a review of the efficacy and safety information available for each.

\section{Hormone therapy}

\section{Estrogen replacement}

Hormone replacement therapy remains the most effective treatment for VMS. According to the most recent Cochrane review, hormone therapy reduces the frequency and severity of hot flashes by $75 \%-79 \% .{ }^{36}$ There are numerous estrogen and estrogen-progestin combination products available for women to use. Studies have shown hormone therapy to be well tolerated, with the most common side effects including breast tenderness, headache, and uterine bleeding.

Although hormone therapy is effective for the treatment of VMS, results from the Women's Health Initiative (WHI) demonstrate that hormone replacement therapy may have negative effects on breast tissue, as its use has been associated with an increased risk of breast cancer. ${ }^{37}$ The WHI also shows that unopposed estrogen therapy is associated with an increased risk of endometrial cancer. ${ }^{37}$ The WHI has also shown that hormone replacement therapy is associated with an increased risk of cardiovascular disease. Since the WHI report, women have become more apprehensive regarding the use of hormone replacement therapy due to these safety concerns. Current recommendations are to use hormone replacement therapy at the lowest effective dose for the shortest duration of time. ${ }^{2}$ Because of these findings and the reaction of women towards them, there has been increased demand for alternative medications to treat VMS.

\section{Tibolone}

Tibolone is another option for the treatment of VMS. It is available in Europe, Canada, and some other countries in Latin America and Asia; it is not approved in the United States. Tibolone is a synthetic drug, and its metabolites have properties similar to estrogen, androgen, and progesterone. ${ }^{38}$ Compared to hormone replacement therapy, studies have found that there is a lower incidence of uterine bleeding with tibolone. ${ }^{38}$ Relative to VMS, most studies show that its efficacy is comparable to hormone replacement therapy. ${ }^{39}$ However, its use carries similar risks to hormone replacement therapy in breast and endometrial tissues. A number of studies have indicated that tibolone may increase the risk of breast cancer and endometrial hyperplasia. ${ }^{38,40,41}$ For these reasons,

Table 2 Herbals and vitamins for the treatment of vasomotor symptoms (VMS) ${ }^{20-35}$

\begin{tabular}{|c|c|}
\hline Herbal/vitamin & Efficacy/safety information \\
\hline Black & $\begin{array}{l}\text { - Two meta-analyses showed black cohosh to have some benefit in treating VMS. }{ }^{20,21} \\
\text { - One randomized trial showed black cohosh to have similar efficacy to tibolone at reducing VMS, as } \\
\text { measured by the Kupperman Menopause Index. }{ }^{22} \\
\text { - One meta-analysis and another randomized controlled trial have shown no benefit to treatment with } \\
\text { black cohosh. }{ }^{22,24} \\
\text { - Long-term effects are unknown, and there exists a possibility of hepatotoxicity. } .^{20,21} \text { However, a recent } \\
\text { meta-analysis of five studies (two had a duration of } 12 \text { weeks, two had a duration of } 16 \text { weeks, and one } \\
\text { had a duration of } 24 \text { weeks) showed that black cohosh does not negatively impact liver function. } .^{25} \\
\text { - The overall long-term efficacy and safety data for black cohosh remain unclear. }\end{array}$ \\
\hline Black cohosh with St John's wort & $\begin{array}{l}\text { - A randomized controlled trial showed that the combination of these herbals significantly improved VMS } \\
\text { and symptoms of depression as measured by the Menopause Rating Scale and Hamilton Depression } \\
\text { Rating Scale, respectively, compared with placebo. }{ }^{26}\end{array}$ \\
\hline Dehydroepiandrosterone (DHEA) & $\begin{array}{l}\text { - A small study has shown a reduction in the frequency of hot flushes in patients taking DHEA supplements. }{ }^{27} \\
\text { - Larger randomized trials are needed to further evaluate these findings. }\end{array}$ \\
\hline Evening primrose oil & - Randomized trials have not shown any benefit in the frequency of hot flushes with evening primrose oil. ${ }^{28}$ \\
\hline Phytoestrogens & - One clinical trial and two meta-analyses do not support the use of soy for vasomotor symptoms. ${ }^{29-31}$ \\
\hline $\begin{array}{l}\text { - Isoflavones } \\
\text { - Soy } \\
\text { - Red clover } \\
\text { - Lignan }\end{array}$ & $\begin{array}{l}\text { - A very recent meta-analysis of } 17 \text { trials found soy isoflavone supplements to be significantly more } \\
\text { effective than placebo at reducing the frequency and severity of hot flashes. }{ }^{32} \\
\text { - A recent meta-analysis and randomized clinical trial concluded there was no impact on the incidence and } \\
\text { severity of hot flushes with red clover. }{ }^{30,33} \\
\text { - Flaxseed, which is very high in lignan, has been studied for the treatment of hot flushes and has been } \\
\text { shown to successfully reduce VMS. }{ }^{34}\end{array}$ \\
\hline Vitamin E & $\begin{array}{l}\text { - A randomized clinical trial showed that Vitamin } \mathrm{E} \text { reduced the occurrence of hot flashes by about one } \\
\text { per day; } ;^{35} \text { however, it was not preferred by patients over placebo. } \\
\text { - Not recommended at this time due to clinically insignificant effects. }\end{array}$ \\
\hline
\end{tabular}


tibolone may not be any safer than hormone replacement therapy itself.

\section{Tissue-selective estrogen complexes}

A new hormone therapy being developed in the US is tissueselective estrogen complexes. This therapy combines a selective estrogen receptor modulator (SERM) with estrogen. SERMs act as agonists or antagonists in different tissues, and each SERM has a distinct profile of action. With the appropriate SERM in place, estrogen's negative effects are blocked in breast and endometrial tissues. This allows the estrogen to impart its positive effects on bone and vaginal tissue as well as reduce VMS. ${ }^{42}$

Currently, the SERM bazedoxifene, which is an agonist in the bone and an antagonist in the breast and uterus, is being studied in combination with estrogen for the treatment of menopausal symptoms. The Phase III SMART-1 (Selective estrogens, Menopause and Response to Therapy) trial demonstrated that the combination of estrogen and bazedoxifene significantly reduces hot flashes at most time points, with higher doses of bazedoxifene being more effective. ${ }^{43}$ The results also show that there is a very low incidence of endometrial hyperplasia after 2 years of treatment. More randomized controlled trials are underway to assess the long-term effect of this combination therapy on the bone and breast. Nevertheless, tissue-selective estrogen complexes have the possibility of providing comparable efficacy to hormone replacement therapy with an improved safety and tolerability profile.

\section{Nonhormone therapies Clonidine}

A number of double-blind, controlled trials have evaluated the efficacy of clonidine for VMS. ${ }^{44,45}$ One trial studied the clonidine patch versus placebo in 110 women with a history of breast cancer and found that clonidine reduces the frequency of hot flashes by $20 \%$ at 4 weeks $(P<0.001) .{ }^{44}$ Another study evaluated 149 postmenopausal women over 8 weeks and found a decrease in hot-flash frequency of $38 \%$ in the clonidine group versus a $24 \%$ reduction in placebo $(P=0.006) .{ }^{45}$ Both of these trials did show that clonidine is associated with considerable adverse effects, including dry mouth, constipation, and drowsiness. ${ }^{44,45}$ Based on a recent meta-analysis, the results of these trials have been rather inconsistent regarding the efficacy of clonidine in VMS. ${ }^{30}$ Half of the trials included in this meta-analysis show that clonidine significantly reduces VMS frequency compared to placebo, while the other half of the trials show no significant difference. With its inconsistent efficacy data and substantial adverse effects, clonidine is not an ideal choice for VMS treatment.

\section{Gabapentin}

Multiple randomized controlled trials evaluating the efficacy of gabapentin for the treatment of VMS show that gabapentin can successfully reduce VMS frequency compared to placebo. ${ }^{46,47}$ One trial involving 59 postmenopausal women compared $900 \mathrm{mg} /$ day gabapentin versus placebo and found a $45 \%$ reduction in the gabapentin group versus $29 \%$ in the placebo group at week $12(P=0.02) .{ }^{46}$ A larger trial involving 420 women with breast cancer evaluated gabapentin $300 \mathrm{mg} /$ day, $900 \mathrm{mg} /$ day, and placebo. ${ }^{47}$ The study showed a $49 \%$ reduction in hot-flash severity scores at week 8 in the $900-\mathrm{mg}$ group, a 33\% reduction in the $300-\mathrm{mg}$ group, and a $21 \%$ reduction in the placebo group $(P=0.007$ for all comparisons). ${ }^{47}$ A randomized control trial of 60 postmenopausal women comparing gabapentin titrated up to $2400 \mathrm{mg} /$ day, estrogen therapy, and placebo showed that gabapentin reduced the frequency of VMS by $71 \%$, compared to $72 \%$ in the estrogen group and $54 \%$ in the placebo group at week 12 ( $P=0.04$ for gabapentin compared to placebo; no significant difference between gabapentin and estrogen). ${ }^{48}$ Larger and longer trials are warranted to further examine the efficacy and safety of gabapentin for treating VMS.

\section{Pregabalin}

Pregabalin is currently indicated for the treatment of fibromyalgia, partial-onset seizures, postherpetic neuralgia, and pain associated with diabetic neuropathy. Chemically, it is similar to gabapentin, and for that reason, its use in treating VMS is being evaluated. ${ }^{49}$ Results of a Phase III randomized trial involving 163 postmenopausal women over 6 weeks demonstrated that pregabalin at doses of $75 \mathrm{mg}$ twice daily and $150 \mathrm{mg}$ twice daily reduced hot-flash scores by $65 \%$ and $71 \%$, respectively, compared to only $50 \%$ in the placebo group $\left(P=0.009\right.$ and $P=0.007$, respectively). ${ }^{50}$ Larger, longer-term trials are needed to confirm these results.

\section{Selective serotonin reuptake inhibitors/ serotonin-norepinephrine reuptake inhibitors}

The selective serotonin reuptake inhibitors (SSRIs), such as citalopram, escitalopram, fluoxetine, paroxetine, and sertraline, and the serotonin-norepinephrine reuptake inhibitors (SNRIs), such as duloxetine, venlafaxine, and desvenlafaxine, have been investigated for the 
treatment of VMS. Two randomized controlled trials have shown that paroxetine reduces symptoms of VMS. ${ }^{51,52}$ In one study, 156 menopausal women received paroxetine $12.5 \mathrm{mg} /$ day, $25 \mathrm{mg} /$ day, or placebo. The study demonstrated a $62 \%$ reduction in hot-flash score at 6 weeks in the $12.5-\mathrm{mg}$ group, a $65 \%$ reduction in the $25-\mathrm{mg}$ group, and a $38 \%$ reduction in the placebo group $(P=0.03$ and $P=0.007$ vs placebo, respectively). ${ }^{51}$ In another study, 151 postmenopausal women received 4 weeks of paroxetine $10 \mathrm{mg}$ /day, paroxetine $20 \mathrm{mg} /$ day, or placebo. Results showed the 10-mg group reduced the hot-flash severity score by $40.6 \%$ compared to the $13.7 \%$ reduction in the placebo group $(P=0.006)$, while the 20 -mg group reduced hot-flash severity score by $51.7 \%$, compared to $26.6 \%$ for the placebo comparator $(P=0.002) .{ }^{52}$ Randomized trials have shown that citalopram, escitalopram, and fluoxetine are also effective at reducing the frequency of hot flashes. In a trial evaluating citalopram, 254 postmenopausal women received citalopram $10 \mathrm{mg} /$ day, citalopram $20 \mathrm{mg} /$ day, citalopram $30 \mathrm{mg} /$ day, or placebo. ${ }^{53}$ After 6 weeks, there was a mean reduction in hot-flash severity score of $49 \%$ in the $10-\mathrm{mg}$ group, $50 \%$ in the 20 -mg group, and $55 \%$ in the $30-\mathrm{mg}$ group compared to $23 \%$ in the placebo group $(P<0.002$ for all comparisons $) .{ }^{53} \mathrm{~A}$ recent randomized double-blind placebo-controlled trial evaluated escitalopram for vasomotor symptoms. The trial enrolled 205 postmenopausal women that were randomized to escitalopram 10-20 mg/day or placebo for 8 weeks. The results showed women in the escitalopram group experienced a significant reduction in hot-flash frequency $(P<0.001)$ and hot-flash severity score $(P<0.001) .{ }^{54}$ In a trial studying fluoxetine, 81 postmenopausal women were randomized to fluoxetine $20 \mathrm{mg} /$ day or placebo for 4 weeks and then crossed over to the other treatment arm. ${ }^{55}$ In the first part of the trial, the $20-\mathrm{mg}$ group reduced hot-flash scores by $50 \%$ compared to only $36 \%$ in the placebo group $(P=0.35)$, but after the crossover the reduction in hot flash scores was significant (24\% vs $19 \%$, respectively, $P=0.02) .{ }^{55}$ Other trials have evaluated the efficacy of sertraline for the treatment of vasomotor symptoms. In one double-blind crossover study evaluating sertraline, 62 breast cancer survivors receiving tamoxifen were randomized to $50 \mathrm{mg}$ sertraline or placebo. ${ }^{56}$ The results showed women taking sertraline for 6 weeks had a significant reduction in hot-flash score compared to placebo $(P=0.03) .{ }^{56}$ Another double-blind crossover study of 102 women found that women experienced five fewer hot flashes per week while taking sertraline $50 \mathrm{mg}$ versus placebo $(P<0.002)$; however, the severity of the hot flashes was not significantly different between sertraline and placebo. ${ }^{57}$ Most of the trials evaluating
SSRIs have been relatively small and short in duration, and larger and longer trials are warranted. Overall, SSRIs appear to be effective in reducing VMS.

One very small trial has evaluated the SNRI duloxetine with favorable outcomes. In an open-label trial, 20 postmenopausal women with major depressive disorder were given duloxetine at $60-120 \mathrm{mg} /$ day. At the end of 8 weeks, the women showed a significant improvement in the Montgomery-Asberg Depression Rating Scale score from baseline $(P<0.001){ }^{58}$ Trials evaluating the SNRI venlafaxine for VMS have yielded positive results, showing an approximate 55\% reduction in VMS. ${ }^{59,60}$ In one study, 191 postmenopausal women were assigned to placebo or to target venlafaxine doses of $37.5 \mathrm{mg} /$ day, $75 \mathrm{mg} /$ day, or $150 \mathrm{mg}$ /day. Hot-flash scores reduced from baseline by $27 \%$ in the placebo group, $37 \%$ in the $37.5-\mathrm{mg}$ group, $61 \%$ in the $75-\mathrm{mg}$ group, and $61 \%$ in the 150 -mg group after 4 weeks $(P<0.0001$ for all comparisons vs placebo). ${ }^{59}$ This study demonstrated that higher doses of venlafaxine did not correlate with improved efficacy; however, the 150-mg dose had significantly more side effects compared to placebo $(P<0.05)$, including dry mouth, decreased appetite, nausea, and constipation. ${ }^{59}$ Another trial of 109 postmenopausal women compared venlafaxine at a dose of $75 \mathrm{mg} /$ day to a single injection of $400 \mathrm{mg}$ of depomedroxyprogesterone acetate (DMPA). ${ }^{60}$ There was a $55 \%$ reduction in hot-flash score in the venlafaxine group, versus a $79 \%$ reduction in the DMPA group after 6 weeks of treatment $(P<0.001) .{ }^{60}$ Although venlafaxine does not appear to be superior to hormone replacement therapy, it is still a good option for the treatment of vasomotor symptoms.

Desvenlafaxine is the major active metabolite of venlafaxine, and therefore it has been postulated that desvenlafaxine may be effective at reducing VMS frequency and severity. In a study that evaluated the effects of desvenlafaxine on temperature regulation in ovariectomized rats, desvenlafaxine significantly increased serotonin and norepinephrine levels in areas of the hypothalamus that are important for temperature homeostasis. ${ }^{61}$ Therefore, it is hypothesized that desvenlafaxine would be effective in treating VMS caused by temperature dysregulation in the hypothalamus, specifically for VMS associated with menopause.

\section{Desvenlafaxine clinical trials}

To date, four clinical trials have evaluated desvenlafaxine's efficacy in treating VMS in postmenopausal women. ${ }^{62-65}$ In Speroff and colleagues, 707 postmenopausal women were assigned to desvenlafaxine $50,100,150$, or $200 \mathrm{mg} /$ day 
or placebo and evaluated over 12 weeks. ${ }^{62}$ In one trial by Archer and colleagues, 484 postmenopausal women received desvenlafaxine $100 \mathrm{mg} /$ day, $150 \mathrm{mg}$ /day, or placebo for 12 weeks. ${ }^{63}$ In another trial conducted by Archer and colleagues, 458 postmenopausal women were assigned to desvenlafaxine $100 \mathrm{mg} /$ day, $150 \mathrm{mg}$ /day, or placebo for 12 weeks. ${ }^{64}$ Finally, in a trial conducted by Bouchard and colleagues, 485 women received desvenlafaxine $100 \mathrm{mg} /$ day, tibolone $2.5 \mathrm{mg} /$ day, or placebo for 12 weeks. ${ }^{65}$ The inclusion and exclusion criteria of the four trials were similar. Women must have been postmenopausal, generally healthy, have a $\mathrm{BMI}<40 \mathrm{~kg} / \mathrm{m}^{2}\left(<34 \mathrm{~kg} / \mathrm{m}^{2}\right.$ for the Bouchard et al trial $\left.{ }^{65}\right)$, and have reported a specific quantity of moderate to severe hot flashes (seven per day or 50 per week). Women who had recently received hormone therapy or any medications thought to treat VMS, having a history of seizure disorder, myocardial infarction, or malignancy, or having hepatic dysfunction, renal dysfunction, a psychiatric disorder, or any other major medical disease were excluded. No significant differences were observed between the treatment groups with regard to the baseline demographics in these trials. The majority of patients in each of these trials were of Caucasian descent (80\%-90\%). Table 3 describes the design, primary and secondary end points, and results for each of these clinical trials.

\section{Efficacy results - primary end points}

The four clinical trials evaluating desvenlafaxine for the treatment of menopause-related VMS used two common primary end points: the change in the daily frequency of moderate-to-severe hot flashes and the change in the daily average hot-flash severity score. ${ }^{62-65}$ In three of the trials, the change in the daily average hot-flash severity score was calculated as: ([number of mild hot flashes $\times 1]+[$ number of moderate not flashes $\times 2]+[$ number of severe hot flashes $\times 3]$ )/total number of hot flashes on that day. ${ }^{62,63,65}$ In the fourth clinical trial, the procedure for calculating the average daily hot-flash severity score was not clearly defined. ${ }^{64}$

In both studies conducted by Archer and colleagues, the $100-\mathrm{mg} /$ day and $150-\mathrm{mg} /$ day doses of desvenlafaxine were superior to placebo at reducing the number of moderate-to-severe hot flashes at weeks 4 and 12.63,64 In these two studies, both desvenlafaxine groups experienced a reduction in the frequency of moderate-to-severe hot flashes by $60 \%-66 \%$. In one Archer trial, the desvenlafaxine 100-mg group and 150-mg group differed significantly from placebo at weeks 4 and $12(P<0.002$ at week 4 for both groups; $P<0.001$ at week 12 for both groups). ${ }^{63}$ In the other Archer trial, the desvenlafaxine 100-mg group and 150-mg group also differed significantly from placebo at weeks 4 and 12 ( $P \leq 0.012$ for both groups at week $4, P<0.005$ for the 100-mg group, and $P<0.012$ for the 150-mg group at week 12). ${ }^{64}$ In the trial conducted by Speroff and colleagues, desvenlafaxine doses of $100 \mathrm{mg} /$ day and $150 \mathrm{mg} /$ day significantly reduced the average daily number of moderateto-severe hot flashes compared with placebo at week 12 by $60 \%-64 \%$ ( $P=0.005$ and $P=0.02$, respectively) ${ }^{62}$ However, in this trial, the results for the desvenlafaxine 150-mg group did not differ significantly from placebo at week 4, and the desvenlafaxine 50-mg and 200-mg groups did not differ significantly from placebo at either week 4 or 12. In contrast, Bouchard and colleagues observed that the average daily number of moderate-to-severe hot flashes was not significantly different for the desvenlafaxine group at weeks 4 and 12 compared to placebo: desvenlafaxine reduced the average number of hot flashes by $57.7 \%$, while placebo reduced the average number of hot flashes by $57.5 \%$ at week $12 .{ }^{65}$ Tibolone reduced the average number of hot flashes by $81 \%$ and differed significantly from placebo at weeks 4 and 12 ( $P<0.001$ for all comparisons $){ }^{65}$

In both studies by Archer and colleagues, the average daily hot-flash severity score differed significantly for both the desvenlafaxine 100-mg and 150-mg groups compared to placebo at weeks 4 and 12 ( $P \leq 0.005$ at both time points; ${ }^{63}$ $P \leq 0.025$ at both time points $\left.{ }^{64}\right)$. In the study by Speroff and colleagues, the 100-mg and 200-mg desvenlafaxine groups differed from placebo at week 12 in terms of the average daily severity score ( $P=0.002$ and $P=0.013$, respectively), but not at week $4 .{ }^{62}$ However, the desvenlafaxine 50-mg and 150 -mg groups were not significantly different from placebo at week 4 or 12 . In the study by Bouchard and colleagues, the average daily hot-flash severity score was not significantly different between the desvenlafaxine 100-mg group and placebo groups at weeks 4 and 12; however, the tibolone 2.5-mg group was significantly different from placebo at both of these time points ( $P<0.001$ for both comparisons) ${ }^{65}$

\section{Efficacy results - secondary endpoints}

Important secondary endpoints of the trials included the proportion of patients achieving a $50 \%$ and $75 \%$ reduction in the number of hot flashes and the number of nighttime awakenings attributed to hot flashes.

The proportion of patients achieving at least a $50 \%$ or $75 \%$ reduction in the number of hot flashes was significantly different between placebo and the 100-mg 


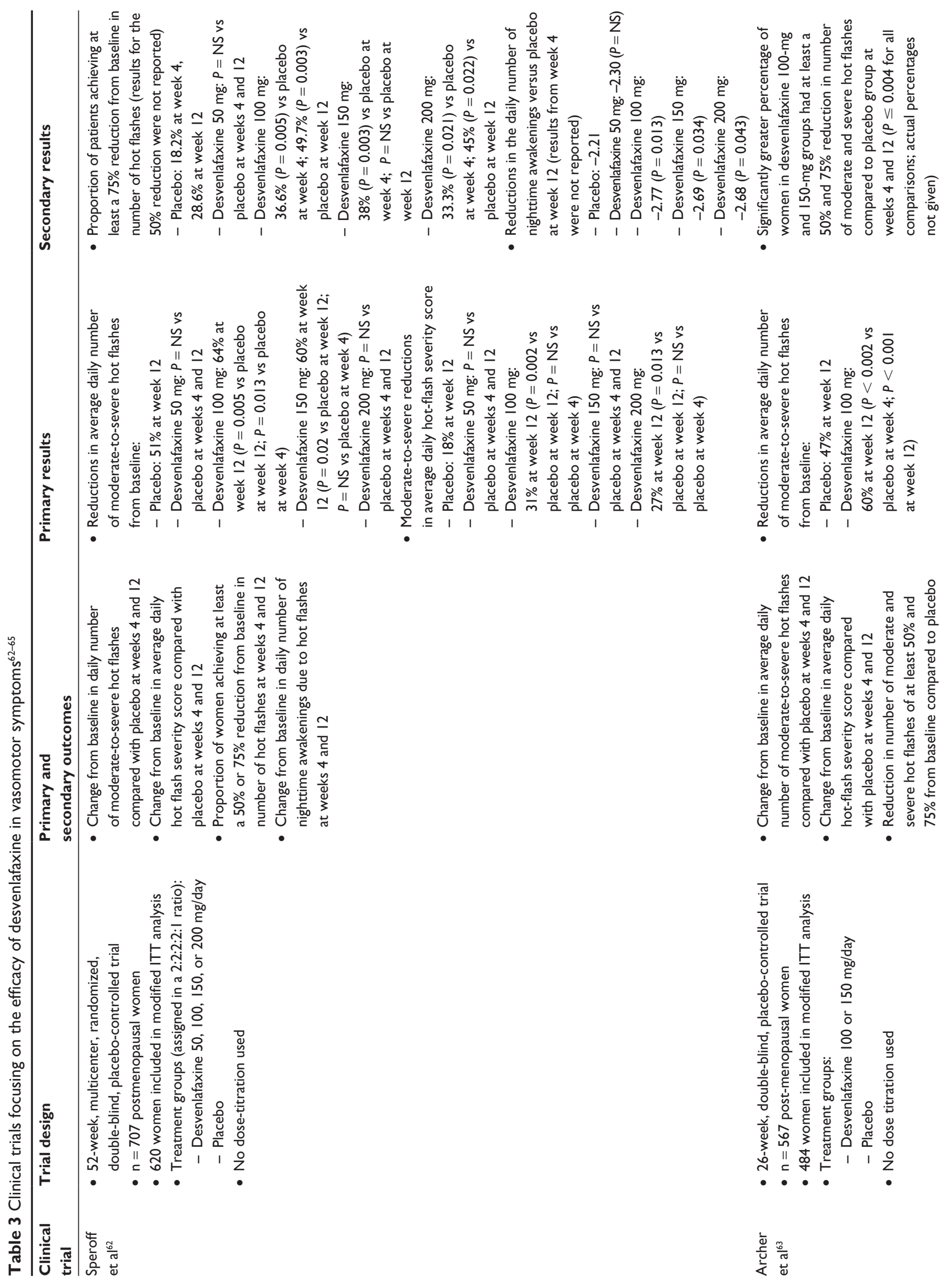



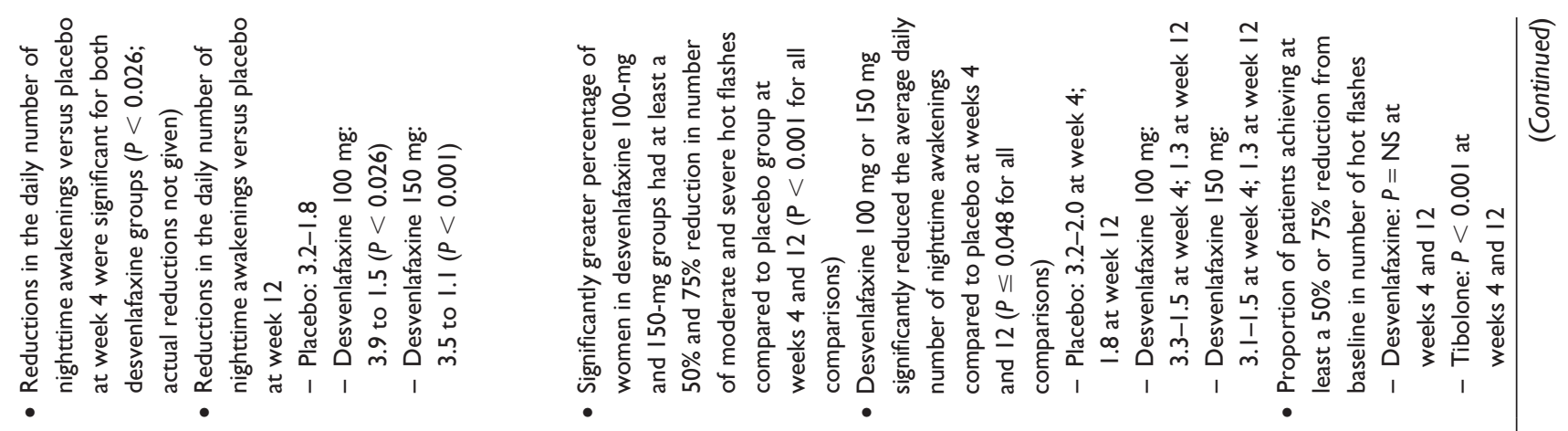

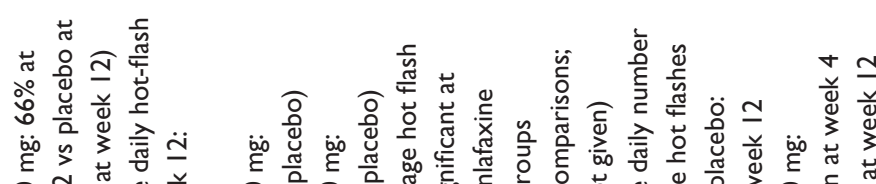

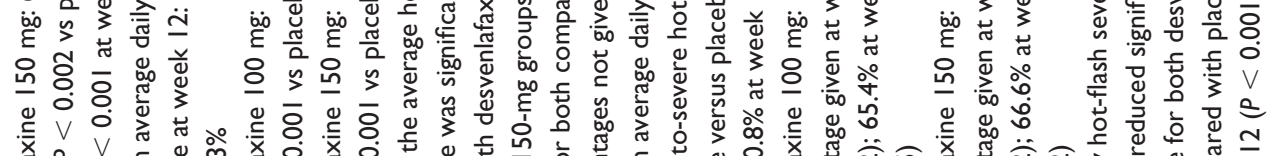

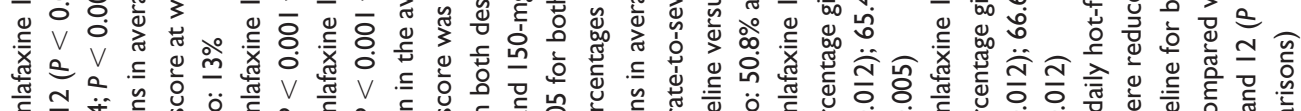

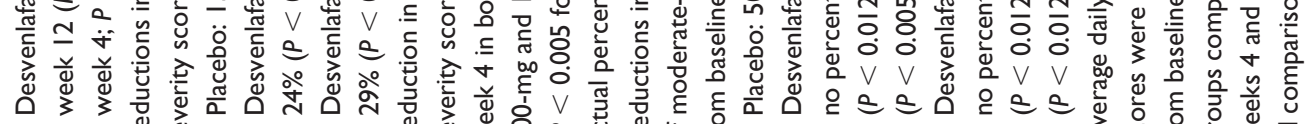
(1)

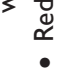
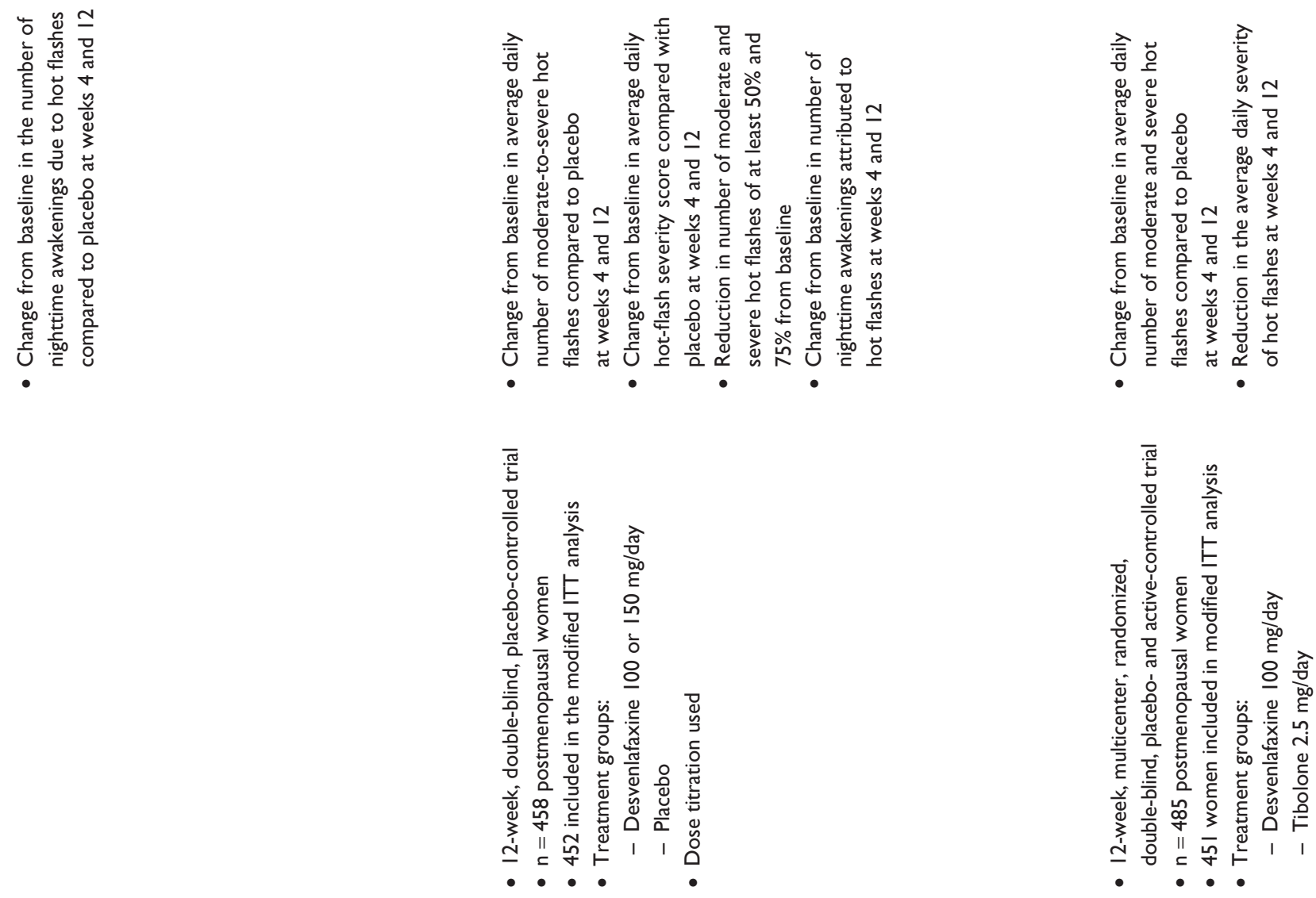

毫焉

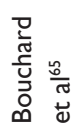


and 150-mg desvenlafaxine groups at weeks 4 and 12 in both trials conducted by Archer and colleagues $(P<0.001$ for all comparisons in one Archer trial; ${ }^{63} P \leq 0.004$ for all comparisons in the other Archer trial ${ }^{64}$ ). Speroff and colleagues observed a significantly greater proportion of patients achieving a $75 \%$ or greater reduction compared with the placebo at week 4 in the desvenlafaxine 100-mg, 150-mg, and 200-mg groups $(P=0.005, P=0.003$, and $P=0.021$, respectively). ${ }^{62}$ At week 12 , only the $100-\mathrm{mg}$ and $200-\mathrm{mg}$ desvenlafaxine groups had significantly greater proportions of patients achieving a 75\% reduction in the number of hot flashes $\left(P=0.003\right.$ and $P=0.022$, respectively).$^{62}$ There was no significant difference between the 50-mg desvenlafaxine group and placebo with regard to this particular end point at week 4 or 12 . The results and statistics for the $50 \%$ reduction were not reported in the trial. In the trial conducted by Bouchard and colleagues, there was no significant difference between the 100-mg desvenlafaxine group and placebo in the proportion of patients achieving at least a $50 \%$ or $75 \%$ reduction in the number of hot flashes at either week 4 or 12 , but there was a significant difference between the 2.5 -mg tibolone group and placebo $(P<0.001$ for all comparisons). ${ }^{65}$

The number of nighttime awakenings attributed to hot flashes was significantly reduced in the desvenlafaxine 100-mg and 150-mg groups compared to placebo at weeks 4 and 12 in both trials by Archer and colleagues $(P<0.026$ and $P<0.001$ at week 4 and $\left.12 ;{ }^{63} P<0.048^{64}\right)$. Speroff and colleagues found that this end point was significantly reduced in the desvenlafaxine 100-mg, 150-mg, and 200-mg groups compared to placebo at week $12(P=0.013, P=0.034$, and $P=0.043$, respectively). ${ }^{62}$ No significant difference in this end point was observed between the desvenlafaxine 50-mg group and placebo at week 12. The results for this end point at week 4 were not reported in this trial. This end point was not evaluated in the trial conducted by Bouchard and colleagues. ${ }^{65}$

\section{Safety and tolerability}

The rates of treatment-emergent adverse effects and discontinuation were also evaluated in the four desvenlafaxine clinical trials. The most commonly observed adverse event in the desvenlafaxine treatment groups in all of these trials was nausea.

The study by Speroff and colleagues found that women receiving desvenlafaxine $150 \mathrm{mg}$ or $200 \mathrm{mg}$ experienced significantly more treatment-emergent adverse events ( $P=0.04$ and $P=0.006$, respectively) than women in 
the placebo group, while treatment-emergent adverse events did not differ significantly from placebo in the desvenlafaxine 50-mg and 100-mg groups. ${ }^{62}$ The incidence of nausea in the trial was around $40 \%-45 \%$ depending on the dose of desvenlafaxine. Discontinuations due to adverse events occurred at significantly higher rates in both the 150-mg and 200-mg desvenlafaxine groups (35\% and $40 \%$, respectively) compared with placebo (both comparisons, $P<0.001$ ), while the rate of discontinuation in the desvenlafaxine 50-mg and 100-mg groups did not significantly differ from that associated with placebo. ${ }^{62}$

In one of the trials by Archer and colleagues, significantly more desvenlafaxine-treated subjects reported treatmentemergent adverse events during week 1 compared to placebo $(P<0.05)$; however, no significant differences between these two groups were observed at any other time point regarding this outcome. ${ }^{63}$ Around $45 \%$ of all patients randomized to desvenlafaxine reported nausea during the trial. Significantly more patients in the desvenlafaxine groups $(26.9 \%$ in the 150 -mg group; $30.2 \%$ in the $200-\mathrm{mg}$ group) discontinued therapy due to treatment-emergent adverse events compared to placebo $(8.9 \% ; P<0.001$ for either treatment compared to placebo). In the second trial by Archer and colleagues, $84.1 \%$ of the patients treated with desvenlafaxine reported treatment-emergent adverse events compared with $69.5 \%$ of patients in the placebo group $(P<0.002) .{ }^{64}$ In this trial $25.5 \%$ of women treated with desvenlafaxine reported nausea during treatment. There were no significant differences in the rates of discontinuation of treatment for any reason, including adverse events, between the desvenlafaxine 100-mg (12.7\%), $150-\mathrm{mg}$ (16.6\%), and placebo groups (9.9\%).

The incidence of uterine bleeding was the primary safety end point in the study conducted by Bouchard and colleagues. ${ }^{65}$ This endpoint was chosen because the comparator drug, tibolone, is a hormonal treatment known to cause uterine bleeding. The incidence of uterine bleeding was significantly higher in the tibolone group (23\%) compared to both the desvenlafaxine and placebo groups $(12 \%, P=0.024$, and $9 \%, P=0.001$, respectively). ${ }^{65}$ Treatment-emergent adverse events were reported among $73.4 \%$ of patients in the $100-\mathrm{mg}$ desvenlafaxine group, $64.5 \%$ of patients in the tibolone group, and $55.9 \%$ of patients in the placebo group ( $P \leq 0.006$ for placebo vs the tibolone and desvenlafaxine groups). Nausea was reported in $8.9 \%$ and $<4 \%$ of patients in the desvenlafaxine and placebo groups, respectively $(P<0.001)$. During the first week of treatment, the rate of drug discontinuation was significantly higher in the desvenlafaxine group (11\%) than in the tibolone $(1.8 \%)$ or placebo $(1.6 \%)$ groups $(P<0.001$ for desvenlafaxine vs the tibolone and placebo groups). The rate of drug discontinuation was not significantly different between the treatment groups at any other time point.

\section{Discussion}

VMS are a disturbing part of menopause for many women and have a significant impact on QOL. Although hormone therapy is the most effective treatment for menopause-related VMS, many women either cannot or do not wish to take this treatment. Therefore, the availability of effective alternative therapies for the treatment of VMS is of great importance. Desvenlafaxine, an SNRI, is one such treatment option for the treatment of VMS in postmenopausal women.

The desvenlafaxine trials published to date have inconsistent efficacy results. Both trials conducted by Archer and colleagues found desvenlafaxine to be more beneficial than placebo in treating VMS in terms of reducing the frequency of moderate-to-severe hot flashes, reducing the average daily hot-flash severity score, and reducing nighttime awakenings at doses of $100 \mathrm{mg}$ and $150 \mathrm{mg} .{ }^{63,64}$ Speroff and colleagues also showed desvenlafaxine to be favorable in treating VMS; however, this trial had some confounding results as not all of the doses were superior to placebo at all identified time points. ${ }^{62}$ Further, while both studies conducted by Archer and the study conducted by Speroff noted the measurement of QOL within their research methods, this data has yet to be made available. ${ }^{62,64}$ Knowing the link between VMS and QOL, ${ }^{3,5}$ the lack of such data is significant. Lastly, Bouchard and colleagues observed no significant difference between the desvenlafaxine and placebo groups with regards to the reduction in the number of moderate-to-severe hot flashes, hotflash severity score, or number of nighttime awakenings. ${ }^{65}$

Several variables may contribute to the difference in efficacy rates observed in the trials. One important factor may be dose titration. Only the one study by Archer and colleagues $^{64}$ titrated the dose of desvenlafaxine (starting all patients on $50 \mathrm{mg} /$ day for at least 3 days, then increasing to $100 \mathrm{mg} /$ day, and finally $150 \mathrm{mg} /$ day over 1 week), while in the other studies patients were started initially on the dose they were randomized to receive $(100 \mathrm{mg}, 150 \mathrm{mg}$, or $200 \mathrm{mg}){ }^{62,64,65}$ Patients not titrated on SNRIs are more likely to experience adverse effects, in particular nausea. ${ }^{66}$ As reported, in three of the four clinical trials presented, ${ }^{62,63,65}$ discontinuation of therapy due to adverse events occurred at significantly higher rates in the desvenlafaxine-treated groups compared to the placebo group. No significant difference in the rate of treatment discontinuation was observed in only one 
of the clinical trials. ${ }^{64}$ This particular trial was the only one to titrate the dose of desvenlafaxine over a week. Further, as all four of these trials used a modified intention-to-treat analysis, having more women discontinuing therapy due to adverse effects could make the desvenlafaxine look less desirable as a treatment option due to safety. In fact, Gallagher and colleagues conducted a study to look at how titrating the desvenlafaxine dose affects patient tolerability. ${ }^{66}$ In this study, patients were randomized to receive desvenlafaxine for 7 days at the following doses: $100 \mathrm{mg} /$ day (with no titration); $50 \mathrm{mg} /$ day (with no titration); $25 \mathrm{mg}$ /day for 4 days titrated to $50 \mathrm{mg}$ /day for 3 days; or $25 \mathrm{mg} /$ day (with no titration). All patients subsequently received desvenlafaxine $100 \mathrm{mg} /$ day for 15 weeks. Patients titrated to desvenlafaxine $100 \mathrm{mg}$ experienced significantly less nausea $(19 \%-25 \%)$ than the desvenlafaxine 100-mg group, whose dose was not titrated (35.2\%; $P<0.05)$. The results of this study suggest that desvenlafaxine should be titrated to avoid unnecessary adverse effects that may lead to discontinuation of therapy. With early discontinuation, the full, potential efficacy of the medication is not able to be properly evaluated.

Another potential factor contributing to the variability of the outcomes in these studies is the population of women included in the analyses. Severity of VMS varies according to time since the onset of menopause. ${ }^{3,4}$ Symptoms are most severe in the 1-4 years surrounding the FMP, with symptoms improving the farther the woman is from her FMP. While the inclusion criteria for the clinical trials were generally similar relative to the frequency and severity of VMS in the included women, ${ }^{62-65}$ and the average age of the women was similar, the age ranges and specifics of the FMP of these women were quite variable. In both studies by Archer and colleagues, the average age in each of the trials was 53 years. ${ }^{63,64}$ In one of the trials, the ages of the women ranged from 29 to 71 years, ${ }^{64}$ although the distribution was not reported. The age distribution was not reported in the other Archer study either. ${ }^{63}$ Similarly, in the Speroff et al trial, the average age was also 53 years; however, the ages ranged from 37 to 78 years old. ${ }^{62}$ Again, the age distribution was not reported in this study. Bouchard and colleagues included postmenopausal women with an average age of 53 years. ${ }^{65}$ As with the other desvenlafaxine trials, no age distribution was reported.

Although the average age of women studied in all of the trials was approximately 53 years, a prominent time for the occurrence of VMS, the age ranges reported were quite vast, with women as young as 29 years and as old as 78 years being included. ${ }^{62-65}$ In particular, for the women of younger ages, whether or not their menopause was surgical was not reported. It is known that women who enter menopause due to surgery tend to experience more severe VMS. ${ }^{3}$ For reasons such as this, it would be very helpful to know the percentages of women within the various age ranges in the trials. Further, it would be helpful to know how many women were surgically menopausal versus naturally menopausal in the 1-4 years since their FMP versus 8 or more years postmenopause. The inclusion of women with widely variable time durations since their FMP may not be prudent to truly evaluate the effects of medications on VMS. Knowledge of this information may help to determine whether the symptom improvement observed was secondary to drug treatment or to the length of time since their FMP. Such variability in the age ranges included in the studies may have been a contributor to the variable findings observed.

Additionally, the trials lacked reporting other information that would be of value in interpreting the trial results and in making clinical recommendations. Important information not reported in these trials relates to the proposed risk factors for VMS. One such determinant, as previously noted, includes whether or not the women had tried another form of therapy (hormone therapy, nonhormone therapy, vitamins/herbals, or even nonpharmacologic) in the past. ${ }^{8}$ Given the proven response rates to hormone therapy for $\mathrm{VMS},{ }^{36}$ women who had previously been on hormone therapy may have been more likely to report an inadequate response to desvenlafaxine in comparison to women who had never tried any kind of therapy before. Although part of the exclusion criteria in the trials was that women could not have been taking any hormone therapy or other medications used for the treatment of VMS within a certain time period before the trial, there was no specific mention of herbals and vitamins as part of this exclusion criteria. As many people do not consider these entities as "medications," the reporting regarding taking these supplements may be inaccurate. If many women were taking herbals and vitamins to treat hot flashes during the studies, this would also be considered a confounding variable.

An interesting factor that may have impacted the results in all of these trials was the placebo effect. In both studies by Archer et al and the study by Speroff et al, the placebo caused a reduction in moderate-to-severe daily hot flashes by about $50 \%{ }^{62-64}$ In the Bouchard et al study, placebo caused a 57.5\% reduction in daily hot flashes. ${ }^{65}$ The reason for the large placebo effect seen in these trials is unknown. Perhaps it could be related to the natural course of the VMS and the age of the women (of note, this is another instance where the distribution of the ages of the women included would be useful); that is, perhaps certain women experienced a natural course of reduction in their VMS 
over time. It may also be an indication of the subjective nature relative to the perception of VMS.

Other limitations of the trials include the study duration, the lack of ethnic diversity, and neglecting to evaluate the presence of VMS risk factors among the study participants. The efficacy endpoints used in all of the trials were evaluated at 12 weeks, which is a rather short duration. As VMS are known to last for many years following the FMP, ${ }^{4}$ longer trials evaluating the efficacy and safety of desvenlafaxine over an extended time are warranted. Finally, as previously mentioned, over $80 \%$ of the patients in all of the trials were Caucasian. This significantly limits the generalizability of these study findings to other races and ethnicities.

\section{Conclusion}

Upon evaluation of the four clinical trials, the results demonstrate that desvenlafaxine is a viable option for the treatment of VMS in postmenopausal women. The results have shown that desvenlafaxine at doses of $100 \mathrm{mg}$ or higher reduces the frequency of moderate-to-severe hot flashes and the number of nighttime awakenings. Further, doses of $100 \mathrm{mg}$ have not been shown to cause significant side effects, as the incidence of treatment-emergent side effects with this dose is comparable to that observed with placebo. ${ }^{62}$ However, while it is a viable option in comparison to other therapies, desvenlafaxine cannot be recommended over the other available treatments. Studies comparing desvenlafaxine to other available treatment options relative to efficacy and safety are lacking, with the exception of the one trial comparing it to tibolone. ${ }^{65}$ The lack of head-to-head trials with the nonhormonal treatments, in particular, leave practitioners with numerous choices, as one medication has not been shown to be superior. Clinical trials comparing desvenlafaxine to other nonhormonal treatments are needed to establish its place relative to these other options. Larger studies with longer durations, inclusive of more diversity among subject ethnicity, and with a focus that includes reporting the impact on overall QOL, are warranted to evaluate the long-term safety and efficacy of desvenlafaxine for VMS treatment and improve the generalizability of the results. Finally, clinical trials evaluating desvenlafaxine should titrate the dose appropriately to minimize the chance of treatment discontinuation, and a more distinct study population should be used that more closely reflects the typical time frame of when VMS are most severe in relation to menopause. At this time, desvenlafaxine is a reasonable option for the treatment of VMS; however, more research is necessary to define its place in therapy.

\section{Acknowledgment}

The authors wish to acknowledge the assistance of Cynthia Sanoski, PharmD, chair of the Department of Pharmacy Practice and associate professor of clinical pharmacy, for her critical review of this manuscript.

\section{Disclosure}

The authors report no conflicts of interest in this work.

\section{References}

1. Soules MR, Sherman S, Parrott E, et al. Executive summary: stages of reproductive aging workshop (STRAW). JWomens Health Gend Based Med. 2001;10(9):843-848.

2. The North American Menopause Society. Estrogen and progestogen use in postmenopausal women: 2010 position statement of the North American Menopause Society. Menopause. 2010;17(2):242-255.

3. Blumel JE, Chedraui P, Baron G, et al. A large multinational study of vasomotor symptom prevalence, duration, and impact on quality of life in middle-age women. Menopause. 2011;18(7):778-785.

4. Politi MC, Schleinitz MD, Col NF. Revisiting the duration of vasomotor symptoms of menopause: a meta-analysis. J Gen Intern Med. 2008;23(9):1507-1513.

5. Thurston RC, Joffe H. Vasomotor symptoms and menopause: findings from the Study of Women's Health across the Nation. Obstet Gynecol. 2011;38:489-501.

6. Gold EB, Colvin A, Avis N, et al. Longitudinal analysis of the association between vasomotor symptoms and race/ethnicity across the menopausal transition: study of women's health across the nation. Am J Public Health. 2006;96(7):1226-1235.

7. Ford K, Sowers M, Crutchfield M, Wilson A, Jannausch M. A longitudinal study of the predictors of prevalence and severity of symptoms commonly associated with menopause. Menopause. 2005; 12(3):308-317.

8. Melby MK, Anderson D, Sievert LL, Obermeyer CM. Methods used in cross-cultural comparisons of vasomotor symptoms and their determinants. Maturitas. 2011;70(2):110-119.

9. Bryant C, Judd FK, Hickey M. Anxiety during the menopausal transition: a systematic review. J Affect Disord. July 21, 2011. [Epub before print.]

10. Deecher DC, Dorries K. Understanding the pathophysiology of vasomotor symptoms (hot flushes and night sweats) that occur in perimenopause, menopause, and postmenopause life stages. Arch Womens Ment Health. 2007;10:247-257.

11. Rossmanith WG, Ruebberdt W. What causes hot flashes? The neuroendocrine origin of vasomotor symptoms in the menopause. Gynecol Endocrinol. 2009;25(5):303-314.

12. Walker EM, Rodriquez AI, Kohn B, et al. Acupuncture versus venlafaxine for management of vasomotor symptoms in patients with hormone receptor positive breast cancer: a randomized, clinical trial. J Clin Oncol. 2010;28(4):634-640.

13. Avis NE, Legault C, Coeytaux RR, et al. A randomized, controlled pilot study of acupuncture treatment for menopausal hot flashes. Menopause. 2008;15(6):1070-1078.

14. Cho SH, Whang WW. Acupuncture for vasomotor menopausal symptoms: a systematic review. Menopause. 2009;16(5):1065-1073.

15. Elkins G, Marcus J, Stearns V, Hasan Rajab M. Pilot evaluation of hypnosis for the treatment of hot flashes in breast cancer survivors. Psychooncology. 2007;16(5):487-492.

16. Elkins G, Marcus J, Stearns V, et al. Randomized trial of a hypnosis intervention for treatment of hot flashes among breast cancer survivors. J Clin Oncol. 2008;26(31):5022-5026.

17. The North American Menopause Society. Treatment of menopauseassociated vasomotor symptoms: position statement of the North American Menopause Society. Menopause. 2004;11(1):11-33. 
18. Daley A, MacArthur C, Mutrie N, et al. Exercise for vasomotor menopausal symptoms. Cochrane Database Syst Rev. 2007;4:CD006108.

19. Thacker HL. Assessing risks and benefits of nonhormonal treatments for vasomotor symptoms in perimenopausal and postmenopausal women. J Womens Health. 2011;20(7):1007-1016.

20. Kronenberg F, Fugh-Berman A. Complementary and alternative medicine for menopausal symptoms: a review of randomized, controlled trials. Ann Intern Med. 2002;137(10):805-813.

21. Shams T, Setia MS, Hemmings R, McCusker J, Sewitch M, Ciampi A. Efficacy of black cohosh-containing preparations on menopausal symptoms: a meta-analysis. Altern Ther Health Med. 2010;16(1):36-44.

22. Bai W, Henneicke-von Zepelin HH, Wang S, et al. Efficacy and tolerability of a medicinal product containing an isopropanolic black cohosh extract in Chinese women with menopausal symptoms: a randomized, double blind, parallel-controlled study versus tibolone. Maturitas. 2007;58(1):31-41.

23. Pockaj BA, Gallagher JG, Loprinzi CL, et al. Phase III double-blind, randomized, placebo-controlled crossover trial of black cohosh in the management of hot flashes: NCCTG Trial N01CC1. J Clin Oncol. 2006; 24(18):2836-2841.

24. Geller SE, Shulman LP, van Breemen RB, et al. Safety and efficacy of black cohosh and red clover for the management of vasomotor symptoms: a randomized controlled trial. Menopause. 2009;16(6):1156-1166.

25. Naser B, Schnitker J, Minkin MJ, de Arriba SG, Nolte KU, Osmers R. Suspected black cohosh hepatotoxicity: no evidence by meta-analysis of randomized controlled clinical trials for isopropanolic black cohosh extract. Menopause. 2011;18(4):366-375.

26. Uebelhack R, Blohmer JU, Graubaum HJ, Busch R, Gruenwald J, Wernecke KD. Black cohosh and St John's wort for climacteric complaints: a randomized trial. Obstet Gynecol. 2006;107:247-255.

27. Stomati M, Monteleone P, Casarosa E, et al. Six-month oral dehydroepiandrosterone supplementation in early and late postmenopause. Gynecol Endocrinol. 2000;14(5):342-363.

28. Chenoy R, Hussain S, Tayob Y, O’Brien PM, Moss MY, Morse PF. Effect of oral gamolenic acid from evening primrose oil on menopausal flushing. BMJ. 1994;308(6927):501-503.

29. St Germain A, Peterson CT, Robinson JG, Alekel DL. Isoflavone-rich or isoflavone-poor soy protein does not reduce menopausal symptoms during 24 weeks of treatment. Menopause. 2001;8(1):17-26.

30. Nelson HD, Vesco KK, Haney E, et al. Nonhormonal therapies for menopausal hot flashes: systematic review and meta-analysis. JAMA. 2006;295(17):2057-2071.

31. Krebs EE, Ensrud KE, MacDonald R, Wilt TJ. Phytoestrogens for treatment of menopausal symptoms: a systematic review. Obstet Gynecol. 2004;104(4):824-836.

32. Taku K, Melby MK, Kronenberg F, Kurzer MS, Messina M. Extracted or synthesized soybean isoflavones reduce menopausal hot flash frequency and severity: systematic review and meta-analysis of randomized controlled trials. Menopause. March 19, 2012. [Epub before print.]

33. Tice JA, Ettinger B, Ensrud K, Wallace R, Blackwell T, Cummings SR. Phytoestrogen supplements for the treatment of hot flashes: the Isoflavone Clover Extract (ICE) Study: a randomized controlled trial. JAMA. 2003;290(2):207-214.

34. Lewis JE, Nickell LA, Thompson LU, Szalai JP, Kiss A, Hilditch JR. A randomized controlled trial of the effect of dietary soy and flaxseed muffins on quality of life and hot flashes during menopause. Menopause. 2006;13(4):631-642.

35. Barton DL, Loprinzi CL, Quella SK, et al. Prospective evaluation of vitamin E for hot flashes in breast cancer survivors. J Clin Oncol. 1998;16(2):495-500.

36. MacLennan AH, Broadbent JL, Lester S, Moore V. Oral oestrogen and combined oestrogen/progestogen therapy versus placebo for hot flushes. Cochrane Database Syst Rev. 2004;4:CD002978.

37. Rossouw JE, Anderson GL, Prentice RL, et al. Risks and benefits of estrogen plus progestin in healthy postmenopausal women: principal results from the Women's Health Initiative randomized controlled trial. JAMA. 2002;288(3):321-333.
38. Livial (tibolone) [package insert]. Cambridge, MA: Organon Laboratories; 2006.

39. Modelska K, Cummings S. Tibolone for postmenopausal women: systematic review of randomized trials. J Clin Endocrinol Metab. 2002; 87(1):16-23.

40. Beral V. Breast cancer and hormone-replacement therapy in the Million Women Study. Lancet. 2003;362(9382):419-427.

41. Kenemans P, Bundred NJ, Foidart JM, et al. Safety and efficacy of tibolone in breast-cancer patients with vasomotor symptoms: a doubleblind, randomised, non-inferiority trial. Lancet Oncol. 2009;10(2): 135-146.

42. Archer DF. Tissue-selective estrogen complexes: a promising option for the comprehensive management of menopausal symptoms. Drugs Aging. 2010;27(7):533-544.

43. Pickar JH, Yeh IT, Bachman G, et al. Endometrial effects of a tissue selective estrogen complex (TSEC) containing bazedoxifene/conjugated estrogens as a menopausal therapy. Fertil Steril. 2009;92(10): 1018-1024.

44. Goldberg RM, Loprinzi CL, O'Fallon JR, et al. Transdermal clonidine for ameliorating tamoxifen-induced hot flashes. J Clin Oncol. 1994;12(1):155-158.

45. Pandya KJ, Raubertas RF, Flynn PJ, et al. Oral clonidine in postmenopausal patients with breast cancer experiencing tamoxifen-induced hot flashes: a University of Rochester Cancer Center Community Clinical Oncology Program study. Ann Intern Med. 2000;132(10):788-793.

46. Guttuso T, Kurlan R, McDermott MP, Kieburtz K. Gabapentin's effects on hot flashes in postmenopausal women: a randomized controlled trial. Obstet Gynecol. 2003;101(2):337-345.

47. Pandya KJ, Morrow GR, Roscoe JA, et al. Gabapentin for hot flashes in 420 women with breast cancer: a randomised double-blind placebocontrolled trial. Lancet. 2005;366(9488):818-824.

48. Reddy SY, Warner H, Guttuso T, et al. Gabapentin, estrogen, and placebo for treating hot flushes: a randomized controlled trial. Obstet Gynecol. 2006;108(1):41-48.

49. Pachman DR, Jones JM, Loprinzi CL, et al. Management of menopauseassociated vasomotor symptoms: current treatment options, challenges and future directions. Int J Women's Health. 2010;2:123-135.

50. Loprinzi CL, Qin R, Balcueva EP, et al. Phase III, randomized, doubleblind, placebo-controlled evaluation of pregabalin for alleviating hot flashes, N07C1. J Clin Oncol. 2010;28(4):641-647.

51. Stearns V, Beebe KL, Lyengar M, Dube E. Paroxetine controlled release in the treatment of menopausal hot flashes: a randomized controlled trial. JAMA. 2003;289(21):2827-2834.

52. Stearns V, Slack R, Greep N, et al. Paroxetine is an effective treatment for hot flashes: results from a prospective randomized clinical trial. J Clin Oncol. 2005;23(28):6919-6930.

53. Barton DL, LaVasseur B, Sloan JA, et al. A phase III trial evaluating three doses of citalopram for hot flashes: NCCTG trial N05C9. J Clin Oncol. 2010;28(20):3278-3283.

54. Freeman EW, Guthrie KA, Caan B, et al. Efficacy of escitalopram for hot flashes in healthy menopausal women: a randomized controlled trial. JAMA. 2011;305(3):267-274.

55. Loprinzi CL, Sloan JA, Perez EA, et al. Phase III evaluation of fluoxetine for treatment of hot flashes. J Clin Oncol. 2002;20(6):1578-1583.

56. Kimmick GG, Lovato J, McQuellon R, Robinson E, Muss HB. Randomized, double-blind, placebo-controlled, crossover study of sertraline (Zoloft) for the treatment of hot flashes in women with early stage breast cancer taking tamoxifen. Breast J. 2006;12(2):114-122.

57. Gordon PR, Kerwin JP, Boesen KG, Senf J. Sertraline to treat hot flashes: a randomized controlled, double-blind, crossover trial in a general population. Menopause. 2006;13(4):568-575.

58. Joffe H, Soares CN, Petrillo LF, et al. Treatment of depression and menopause-related symptoms with the serotonin-norepinephrine reuptake inhibitor duloxetine. J Clin Psychiatry. 2007;68(6):943-950.

59. Loprinzi CL, Kugler JW, Sloan JA, et al. Venlafaxine in management of hot flashes in survivors of breast cancer: a randomised controlled trial. Lancet. 2000;356(9247):2059-2063. 
60. Loprinzi CL, Levitt R, Barton D, et al. Phase III comparison of depomedroxyprogesterone acetate to venlafaxine for managing hot flashes: North Central Cancer Treatment Group Trial N99C7. J Clin Oncol. 2006;24(9):1409-1414.

61. Deecher DC, Alfinito PD, Leventhal L. Alleviation of thermoregulatory dysfunction with the new serotonin and norepinephrine reuptake inhibitor desvenlafaxine succinate in ovariectomized rodent models. Endocrinology. 2007;148(3):1376-1383.

62. Speroff L, Gass M, Constantine G, Olivier S. Efficacy and tolerability of desvenlafaxine succinate treatment for menopausal vasomotor symptoms: a randomized controlled trial. Obstet Gynecol. 2008;111(1): 77-87.

63. Archer DF, Dupont CM, Constantine GD, Pickar JH, Olivier S. Desvenlafaxine for the treatment of vasomotor symptoms associated with menopause: a double-blind, randomized, placebo-controlled trial of efficacy and safety. Am J Obstet Gynecol. 2009;200(3): 238. e1-238. e10.
64. Archer DF, Seidman L, Constantine GD, Pickar JH, Olivier S. A doubleblind, randomly assigned, placebo-controlled study of desvenlafaxine efficacy and safety for the treatment of vasomotor symptoms associated with menopause. Am J Obstet Gynecol. 2009;200(2):172. e1-172. e10.

65. Bouchard P, Panay N, de Villiers TJ, et al. Randomized placebo- and active-controlled study of desvenlafaxine for menopausal vasomotor symptoms. Climacteric. 2012;15(1):12-20.

66. Gallagher JC, Strzinek RA, Cheng RF, Ausmanas MK, Astl D, Seljan P. The effect of dose titration and dose tapering on the tolerability of desvenlafaxine in women with vasomotor symptoms associated with menopause. J Womens Health. 2012;21(2):188-198.
International Journal of Women's Health

\section{Publish your work in this journal}

The International Journal of Women's Health is an international, peerreviewed open-access journal publishing original research, reports, reviews and commentaries on all aspects of women's healthcare including gynecology, obstetrics, and breast cancer. Subject areas include: Chronic conditions (migraine headaches, arthritis, osteoporosis);

\section{Dovepress}

Endocrine and autoimmune syndromes; Sexual and reproductive health; Psychological and psychosocial conditions. The manuscript management system is completely online and includes a very quick and fair peer-review system. Visit http://www.dovepress.com/ testimonials.php to read real quotes from published authors.

Submit your manuscript here: http://www.dovepress.com/international-journal-of-womens-health-journal 